\title{
Signaling Cost Evaluation of SIGMA
}

\author{
Shaojian Fu \\ Mohammed Atiquzzaman \\ School of Computer Science, \\ University of Oklahoma, \\ Norman, OK 73019-6151.
}

\author{
William Ivancic \\ Satellite Networks \& Architectures Branch, \\ NASA Glenn Research Center, \\ 21000 Brookpark Rd. MS 54-8, \\ Cleveland, OH 44135.
}

\begin{abstract}
In our earlier study, we proposed SIGMA, a

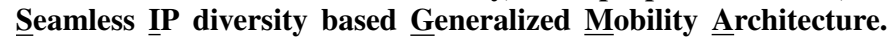
SIGMA utilizes IP diversity to achieve a seamless handover of a mobile host, and is designed to solve many of the drawbacks of Mobile IP. In this paper, we evaluate the signaling cost of SIGMA by using an analytical model. The signaling cost of SIGMA is also compared with Hierarchical Mobile IPv6 (HMIPv6) by using the proposed model. Various aspects that affect signaling cost, such as mobile host moving speed, number of mobile host, number of correspondent node, per-hop transmission cost, and session to mobility ratio, are considered in the analytical model.
\end{abstract}

Keywords: signaling cost, SIGMA, mobile handover

\section{INTRODUCTION}

Mobile IP (MIP) [1] is the standard proposed by IETF to handle mobility of Internet hosts for mobile data communication. Several drawbacks exist when using MIP in a mobile computing environment, the most important ones identified to date are high handover latency, and high packet loss rate [2]. Even with various recent proposed enhancements [2][4], Mobile IP still can not completely remove the handover latency, and the resulting packet loss rate is still high [5].

We designed a new scheme for supporting low latency, low packet loss mobility management called Seamless IP diversity based Generalized Mobility Architecture (SIGMA) [6]. It can cooperate with normal IPv4 or IPv6 infrastructure without the support of Mobile IP. The basic idea of SIGMA is to exploit IP diversity (multihoming) to keep the old path alive during the process of setting up the new path to achieve a seamless handover. SIGMA relies on the signaling message exchange between the $\mathrm{MH}$, correspondent node $(\mathrm{CN})$, and location manager (LM). For every handover, MH sends binding update and location update to CN and LM, respectively. For SIGMA to be useful in real-world wireless system, these signaling messages should not cost too much network bandwidth and should leave enough bandwidth for payload data transmission.

The signaling cost analysis for MIP protocols are presented in [7], [8], but not much work has been done in analyzing the signaling cost of transport layer mobility solutions. The objective of this paper is to look into the signaling cost of SIGMA. Since multihoming is a built-in feature of SCTP, similar to [6], we illustrate SIGMA using SCTP.

The contributions of our paper can be outlined as follows:

- Developed an analytical model for SIGMA signaling cost. 2922.
- Evaluate the signaling cost of SIGMA under various parameters such as mobile host moving speed, number of mobile hosts, number of correspondent nodes, and perhop transmission cost.

The rest of this paper is structured as follows. The necessary preparation work for developing the analytical model is described in Sec. II. The analytical model for S IGMA signaling cost is presented in Sec. III. Then we use the model to evaluate the signaling cost of SIGMA under various input parameters in Sec. IV. Finally, concluding remarks are presented in Sec. V.

\section{Modelling PREPARATION}

In this section, we describe some necessary preparation work for developing an analytical model for SIGMA signaling cost. First, the network structure, the model's assumptions, and notations are presented in Secs. II-A, II-B and II-C, respectively. Then the MH mobility model and traffic arrival model used by signaling cost analysis are set up in Secs. II-D and II-E, respectively. After these modeling foundations are ready, Sec. III develops the signaling cost for location update, binding update and packet delivery in SIGMA.

\section{A. Network structure}

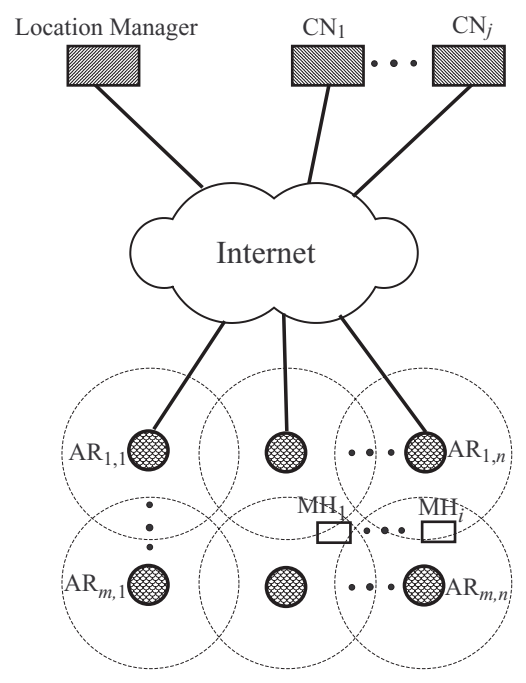

Fig. 1. Network structure considered.

The network structure that will be used in our analytical model is shown in Fig. 1. In the figure, a two dimensional 
subnet arrangement is assumed for modeling $\mathrm{MH}$ movement. $\mathrm{AR}_{1,1}, \cdots \mathrm{AR}_{m, n}$ stand for the access routers. There is a location manager and a number of $\mathrm{CNs}$ connected to the topology through the Internet. The MHs roam around in the subnets covered by $\mathrm{AR}_{1,1}, \cdots \mathrm{AR}_{m, n}$, and each of them communicate with one or more of the CNs. Between a pair of $\mathrm{MH}$ and $\mathrm{CN}$, intermittent file transfers occur due to mobile user requesting information from $\mathrm{CNs}$ using protocols like HTTP. We call each active transfer period during the whole $\mathrm{MH}-\mathrm{CN}$ interactivity as a session.

\section{B. Model assumptions}

We make the following assumptions for developing our analytical model of SIGMA signaling cost.

- In a previous analytical model of P-MIP signaling cost analytical model [8], the session time was assumed to be Pareto distributed and the session arrival was assumed to be Poisson distributed. In our modeling process, both session time and session interval time are Pareto distributed to better model HTTP traffic [9], [10], which is dominant in current Internet traffic load. The Pareto distribution is a heavy-tailed distribution, and it can be characterized with two parameters: minimum possible value $(\kappa)$, and a heavy-tailness factor $(\sigma)$.

- Mobile host moves according to Random Waypoint model [11], which is the most frequently used model in mobile networking research. In this mobility model, an MH randomly selects a destination point in the topology area according to the uniform distribution, then moves towards this point at a random speed again uniformly selected between $\left(v_{\min }, v_{\max }\right)$. One such movement is called an epoch, and the elapsed time and the distance moved during an epoch are called epoch time and epoch length, respectively. At destination point, the $\mathrm{MH}$ will stay stationary for a period of time, called pause time, after which a new epoch starts.

- As in previous studies [7], [8], [12], processing costs at the endpoints ( $\mathrm{MH}$ and $\mathrm{CN}$ ) are not counted into the total signaling cost, since these costs stand for the load that can be scattered into user terminals. Because we are more concerned about the load on the network elements, this assumption enables us to concentrate on the impact of protocol on the network performance.

\section{Notations}

The notations used in this paper are given below.

$N_{m h}$ total number of MHs.

$N_{c n}$ average number of CNs with which a $\mathrm{MH}$ is communicating.

$L U_{m l}$ transmission cost of a location update from $\mathrm{MH}$ to location manager.

$\Psi_{L U}$ location update cost per second for the whole system, including transmission cost and processing cost incurred by location update of all MHs.

$B U_{m c}$ transmission cost of a binding update between $\mathrm{MH}$ and $\mathrm{CN}$.
$\Psi_{B U}$ binding update cost per second between MHs and CNs for the whole system.

$\Psi_{P D}$ packet delivery cost per second from $\mathrm{CNs}$ to $\mathrm{MHs}$ for the whole system.

$\Psi_{T O T}$ total signaling cost per second for the whole system. epoch.

$T_{r} \quad$ MH residence time in a subnet.

$T_{s}, T_{i}$ session time and session interval time.

$\kappa_{s}, \kappa_{i}$ minimum session time and session interval time.

$\sigma_{s}, \sigma_{i}$ heavy-tailness factor for session time and for session interval time.

\section{Mobility model}

The objective of this section is to find the average residence time $\left(T_{r}\right)$ for $\mathrm{MH}$ in a subnet, which gives us the frequency with which the $\mathrm{MH}$ changes its point of attachment, i.e. the frequency of updating LM and CN. $T_{r}$ can be estimated by the time between two successive movements (epoch time $E[T]$ plus pause time $E[P]$ ) divided by the number of subnet crossing during this epoch $E[C]$, as shown in Eqn. (1):

$$
T_{r}=\frac{E(T)+E(P)}{E(C)}
$$

We first compute expected value of epoch time $E[T]$. Since epoch length $L$ and $\mathrm{MH}$ moving speed $v$ are independent:

$$
E[T]=E(L / v)=E(L) E(1 / v)
$$

Since the moving speed is of uniform distribution between $\left(v_{\min }, v_{\max }\right)$, we have:

$$
\begin{aligned}
E[1 / v] & =\int_{v_{\min }}^{v_{\max }}(1 / v) \frac{1}{v_{\max }-v_{\min }} d v \\
& =\frac{\ln \left(v_{\max } / v_{\min }\right)}{v_{\max }-v_{\min }}
\end{aligned}
$$

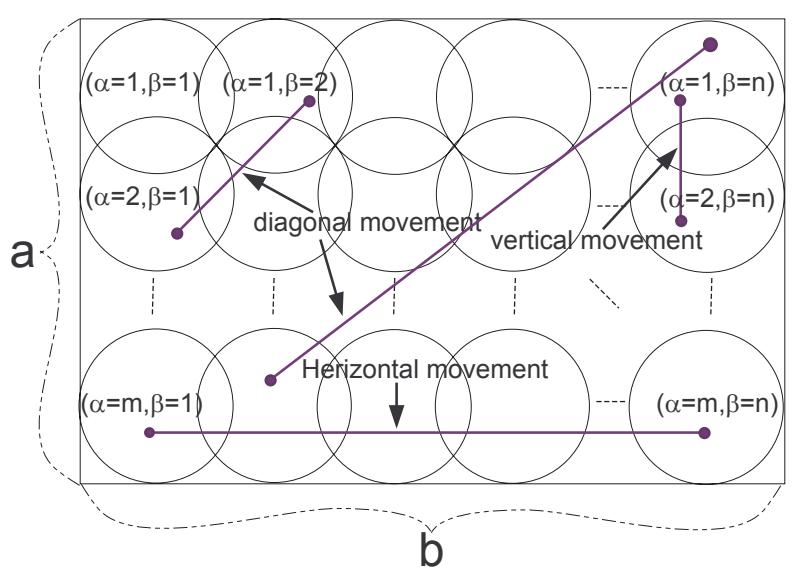

Fig. 2. Arrangement of subnets in a rectangular topology

In order to determine $E(L)$ and $E(C)$, we assume an arrangement of circular subnets in a rectangular topology as shown in Fig. 2, where $m$ and $n$ are the number of vertically and horizontally arranged subnets in the topology, respectively. 
From [11], we know that $E(L)$ for a rectangular area of size $a \times b$ can be estimated as:

$$
\begin{gathered}
E[L]=\frac{1}{15}\left[\frac{a^{3}}{b^{2}}+\frac{b^{3}}{a^{2}}+\sqrt{a^{2}+b^{2}}\left(3-\frac{a^{2}}{b^{2}}-\frac{b^{2}}{a^{2}}\right)\right] \\
+\quad \frac{1}{6}\left[\frac{b^{2}}{a} \Phi\left(\frac{\sqrt{a^{2}+b^{2}}}{b}\right)+\frac{a^{2}}{b} \Phi\left(\frac{\sqrt{a^{2}+b^{2}}}{a}\right)\right]
\end{gathered}
$$

where $\Phi(\cdot)=\ln \left(\cdot+\sqrt{(\cdot)^{2}-1}\right)$.

Now we can get $E(T)$ by combining Eqns. (2), (3) and (4). Since pause time has been assumed to be uniform distributed between $\left(0, P_{\max }\right)$, we have:

$$
E[P]=\int_{0}^{P_{\max }} \frac{P}{P_{\max }} d P=P_{\max } / 2
$$

Next, we need to find $E(C)$, the general form of which can be expressed as [11]:

$$
E[C]=\frac{1}{m^{2} n^{2}} \sum_{\alpha_{j}=1}^{m} \sum_{\beta_{j}=1}^{n} \sum_{\alpha_{i}=1}^{m} \sum_{\beta_{i}=1}^{n} C\left(\begin{array}{c}
\left(\alpha_{i}, \beta_{i}\right) \\
\left(\alpha_{j}, \beta_{j}\right)
\end{array}\right)
$$

The value $C\left(\begin{array}{c}\left(\alpha_{i}, \beta_{i}\right) \\ \left(\alpha_{j}, \beta_{j}\right)\end{array}\right)$ is the number of subnet crossings caused by a movement between subnet $\left(\alpha_{i}, \beta_{i}\right)$ to $\left(\alpha_{j}, \beta_{j}\right)$, which depends on the actual subnet shape and arrangement. In the circular subnet arrangement shown in Fig. 2, we can observe three types of movements: horizontal, vertical and diagonal. $C\left(\begin{array}{c}\left(\alpha_{i}, \beta_{i}\right) \\ \left(\alpha_{j}, \beta_{j}\right)\end{array}\right)$ can be generalized by the following Manhattan distance metric:

$$
C\left(\begin{array}{c}
\left(\alpha_{i}, \beta_{i}\right) \\
\left(\alpha_{j}, \beta_{j}\right)
\end{array}\right)=\left|\alpha_{i}-\alpha_{j}\right|+\left|\beta_{i}-\beta_{j}\right|
$$

By substituting Eqn. (7) into Eqn. (6), we can get the expression for $E(C)$ :

$$
E[C]=\frac{1}{m^{2} n^{2}} \sum_{\alpha_{j}=1}^{m} \sum_{\beta_{j}=1}^{n} \sum_{\alpha_{i}=1}^{m} \sum_{\beta_{i}=1}^{n}\left(\left|\alpha_{i}-\alpha_{j}\right|+\left|\beta_{i}-\beta_{j}\right|\right)
$$

Substituting Eqns. (2), (5) and (8) into Eqn. (1), we can get the expression for $T_{r}$.

\section{E. Arrival traffic model}

The objective of this section is to find the average session arrival rate $\left(\lambda_{s} a\right)$. As discussed in Sec. II-B, both session time and session interval time are of Pareto distribution. The PDF function of session time's distribution is [9]:

$$
f_{T_{s}}(t)=\frac{\sigma_{s} \kappa_{s}^{\sigma_{s}}}{t^{\left(\sigma_{s}+1\right)}}
$$

where $\sigma_{s}=1.2$, and $\kappa_{s}$ can be estimated as:

$$
\kappa_{s}=\frac{10 \mathrm{~KB}}{B W_{m c}}+l_{m c} D_{p q}
$$

where $B W_{m c}$ is the bottleneck bandwidth between $\mathrm{CN}$ and MH. Also from [9], we know that the session interval time has a PDF function of:

$$
f_{T_{i}}(t)=\frac{\sigma_{i} \kappa_{i}^{\sigma_{i}}}{t^{\left(\sigma_{i}+1\right)}}
$$

where $\sigma_{i}=1.5$, and $\kappa_{i}=30 \mathrm{~s}$.

Consider $k(k>0)$ consecutive user session arrivals (the start of the session $k+1$ means the end of the session $k$ plus an interval time) as shown in Fig. 3. The total time for $k$ sessions can be calculated as:

$$
T_{t o t}=k\left(T_{s}+T_{i}\right)
$$

So, the session arrival rate is:

$$
\lambda_{s a}=\frac{k}{E\left(T_{t o t}\right)}=\frac{1}{E\left(T_{s}\right)+E\left(T_{i}\right)}
$$

From probability theory, since $T_{s}>1$ and $T_{i}>1$, the expected value of $T_{s}$ and $T_{i}$ are:

$$
\begin{aligned}
& E\left[T_{s}\right]=\int_{0}^{\infty} t f_{T_{s}}(t) d t=\frac{\kappa_{s} \sigma_{s}}{\sigma_{s}-1} \\
& E\left[T_{i}\right]=\int_{0}^{\infty} t f_{T_{i}}(t) d t=\frac{\kappa_{i} \sigma_{i}}{\sigma_{i}-1}
\end{aligned}
$$

By substituting Eqns. (14) and (15) into Eqn. (13), we can get the average session arrival rate.

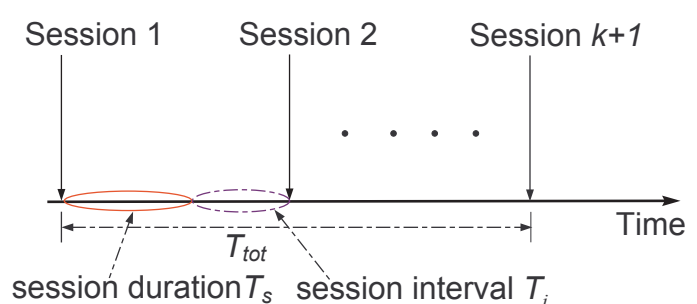

Fig. 3. Illustration of session arrival.

\section{Signaling COST ANALYSis OF SIGMA}

In this section, the signaling cost of SIGMA is analyzed. Subsections III-A, III-B, and III-C develop the cost for location update, binding update and packet delivery, respectively. Finally, subsection III-D gives the total signaling cost of SIGMA.

\section{A. Location update cost}

In SIGMA, every subnet crossing (happens every $T_{r}$ seconds) by an $\mathrm{MH}$ will trigger a location update, which incurs a transmission cost $\left(L U_{m l}\right)$ and processing cost $\left(\gamma_{l}\right)$ for the location update message. Since there is only one location update per subnet crossing, no matter how many $\mathrm{CNs}$ an $\mathrm{MH}$ is communicating with, the number of $\mathrm{CNs}$ does not have any impact on the location update cost. Therefore, the average location update cost per second in the system can be estimated as the number of MHs multiplied by the location update cost for each $\mathrm{MH}$, divided by the average subnet residence time:

$$
\Psi_{L U}^{T}=N_{m h} \frac{L U_{m l}+\gamma_{l}}{T_{r}}
$$

Due to frame retransmissions and medium access contentions at the data link layer of wireless links, transmission cost of a wireless hop is higher than that of a wired hop; we denote this effect by a proportionality constant, $\rho$. Let the 
average distance between $\mathrm{MH}$ and location manager be $l_{m l}$ hops, per-hop location update transmission cost be $\delta_{U}$, for a round trip, $L U_{m l}$ can be calculated as:

$$
L U_{m l}=2\left(l_{m l}-1+\rho\right) \delta_{U}
$$

where $\left(l_{m l}-1\right)$ represents the number of wired hops. Therefore,

$$
\Psi_{L U}^{T}=N_{m h} \frac{2\left(l_{m l}-1+\rho\right) \delta_{U}+\gamma_{l}}{T_{r}}
$$

\section{B. Binding update cost}

In the analysis of binding update cost, processing costs at the endpoints $(\mathrm{MH}$ and $\mathrm{CN}$ ) are not counted into the total signaling cost, since these costs stand for the load that can be scattered into user terminals and hence do not contribute to the network load [7], [8], [12].

Similar to the analysis in Sec. III-A, every subnet crossing triggers a binding update to $\mathrm{CN}$, which incurs a transmission cost $\left(B U_{m c}\right)$ due to the binding update message. For each $\mathrm{CN}$ communicating with an $\mathrm{MH}$, the $\mathrm{MH}$ sends a binding update after each handover. Therefore, the average binding update cost can be estimated as:

$$
\Psi_{B U}^{T}=N_{m h} N_{c n} \frac{B U_{m c}}{T_{r}}
$$

Let the average distance between $\mathrm{MH}$ and $\mathrm{CN}$ be $l_{m c}$ hops, and the per-hop binding update transmission cost be $\delta_{B}$. The $B U_{m c}$ can be calculated as:

$$
B U_{m c}=2\left(l_{m c}-1+\rho\right) \delta_{B}
$$

Therefore, the binding update cost per second in the system can be calculated by multiplying the number of $\mathrm{MHs}$, the average number of communicating $\mathrm{CNs}$, and the average cost per binding update:

$$
\Psi_{B U}^{T}=N_{m h} N_{c n} \frac{2\left(l_{m c}-1+\rho\right) \delta_{B}}{T_{r}}
$$

\section{Packet delivery cost}

Unlike the analysis of packet delivery cost in [7], we do not consider the data packet transmission cost, IP routing table searching cost, and bandwidth allocation cost since these costs are incurred by standard IP switching, which are not particularly related to mobility protocols. Instead, we only consider the location database lookup cost at LM. Moreover we take into account the processing cost caused by packet tunnelling to better reflect the impact of mobility protocol on overall network load.

For SIGMA, a location database lookup at LM is required when an association is being setup between $\mathrm{CN}$ and $\mathrm{MH}$. If each session duration time is independent from each other, the association setup event happens every $S / \lambda_{s a}$ seconds. If we assume the database lookup cost has a linear relationship with $N_{m h}$, and $\varphi_{l}$ and $\psi$ be the per location database lookup cost and the linear coefficient at LM, respectively, then the per-second per-association lookup cost $v_{l}$ can be calculated as:

$$
v_{l}=\frac{\varphi_{l} \lambda_{s a}}{S}=\frac{\psi N_{m h} \lambda_{s a}}{S}
$$

Since SIGMA is free of packet encapsulation or decapsulation, there is no processing cost at intermediate routers. So the packet delivery cost from $\mathrm{CN}$ to $\mathrm{MH}$ can be calculated by only counting the location database lookup cost, which can be expressed as:

$$
\begin{aligned}
\Psi_{P D}^{T} & =N_{m h} N_{c n} v_{l} \\
& =N_{m h}^{2} N_{c n} \frac{\psi \lambda_{s a}}{S}
\end{aligned}
$$

\section{Total signaling cost of SIGMA}

Based on above analysis of the location update cost, binding update cost, and packet delivery cost as shown in Eqns. (18), (21), and (23), the total signaling cost of SIGMA is given by:

$$
\Psi_{T O T}^{T}=\Psi_{L U}^{T}+\Psi_{B U}^{T}+\Psi_{P D}^{T}
$$

\section{RESULTS FROM PROPOSED ANALYTICAL MODEL}

In this section, we present comparison results showing the effect of various input parameters on SIGMA's total signaling cost. The detailed signaling cost analysis of HMIPv6 using the mobility and traffic model described in Sec. II is not presented here in this paper due to space limitation. Readers can refer to [13] for more details.

In all the numerical examples, we use the following parameter values, which are obtained from previous work [7] and our calculation based on user traffic model of Corvella et al. [9] and mobility model of Bettstetter et al. [11]: $\gamma_{l}=30$, $\psi=0.3, F=10 \mathrm{Kbytes}, P M T U=576$ bytes, $S=10$, $\rho=10, l_{m l}=35, l_{m c}=35, m=10, n=8, \gamma_{h}=30$, $\gamma_{m}=20, \tau=0.5, \lambda_{s a}=0.01$.

\section{A. Impact of number of MHs under different maximum $M H$} moving speeds

The impact of number of MHs on total signaling cost of SIGMA and HMIPv6 for different MH moving speed is shown in Fig. 4(a), for $N_{c n}=1$ and $\delta_{U}=\delta_{B}=0.2$. From the figure, we can see that for different moving speeds, the signaling cost of both SIGMA and HMIPv6 increases with an increase in the number of MHs. When the moving speed is high, the subnet residence time $T_{r}$ decreases (see Eqns. (1) , (2), and (3)), resulting in an increase in the location update and binding update costs per second (see Eqns. (18) and (21)). We can also observe that the total signaling cost of SIGMA is less than HMIPv6 in this scenario; this is because when $\delta_{U}$ and $\delta_{B}$ are small, the location update and binding update costs are not high, and the high packet delivery cost makes the signaling cost of HMIPv6 much higher than that of SIGMA.

\section{B. Impact of average number of communicating $C N$ and location update transmission cost}

Next, we set subnet residence time $T_{r}=60 \mathrm{~s}$, and number of MHs $N_{m h}=80$. The impact of the average number of average $\mathrm{CNs}$ with which an $\mathrm{MH}$ communicates with for different perhop transmission cost on location update cost $\left(\delta_{U}\right)$ is shown in Fig. 4(b). It can be observed from this figure that when the average number of communicating $\mathrm{CNs}$ increases, the 


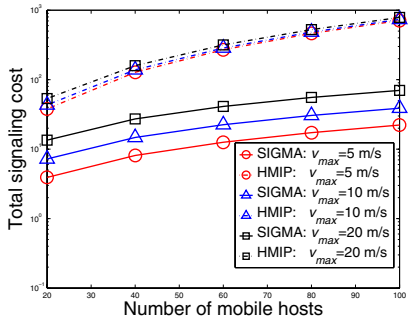

(a) Impact of $N_{m h}$ under different moving speeds

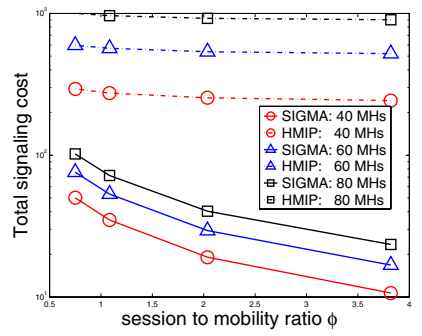

(c) Impact of SMR on total signaling cost under different $N_{m h}$

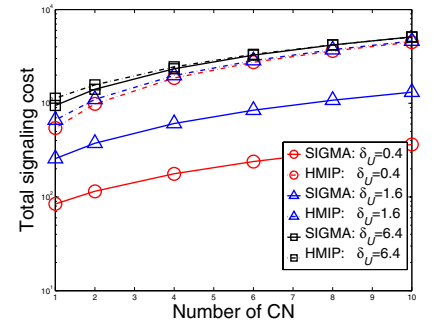

(b) Impact of $N_{C N}$ and per-hop binding update transmission cost

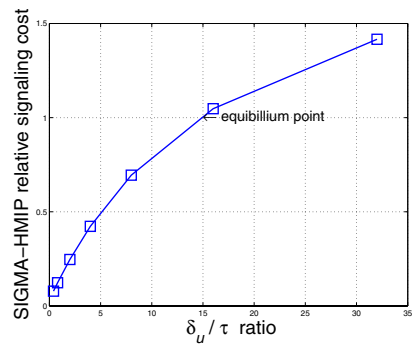

(d) Impact of $\delta_{U} / \tau$ ratio on SIGMA to HMIPv6 relative signaling cost
Fig. 4. Signaling cost comparison of SIGMA and HMIPv6

total signaling cost increases (see Eqns. (18), (21) and (23)). Also, when $\delta_{U}$ increases, the location update cost per second will increase as indicated by Eqn. (17), which will result in an increase in the total signaling cost of both SIGMA and HMIPv6. However, we can see that the impact of $\delta_{U}$ is much smaller in HMIPv6; this is because HMIPv6's signaling cost is less sensitive to location update cost due to its hierarchical structure. In this scenario, signaling cost of HMIPv6 is higher than that of SIGMA when $\delta_{U}=0.4$ or 1.6. However, when $\delta_{U}=6.4$, SIGMA requires a higher signaling cost due to frequent location update for each subnet crossing (compared to HMIPv6's hierarchical mobility management policy).

\section{Session to Mobility Ratio}

Session to Mobility Ratio $(S M R)$ is a mobile packet network's counterpart of Call to Mobility Ratio (CMR) in PCS networks. We vary $T_{r}$ from 75 to 375 seconds with $\lambda_{s a}$ fixed to 0.01 , which yields an $S M R$ of 0.75 to 3.75 . The impact of $S M R$ on total signaling cost for different $N_{m h}$ is shown in Fig. 4(c). We can observe that a higher SMR results in lower signaling cost for both SIGMA and HMIPv6. This is mainly because high $S M R$ means lower mobility, and thus lower signaling cost due to less location and binding updates. Also, we can see that the decrease in HMIPv6's signaling cost as a function of SMR is not as fast as that of SIGMA. This, again, is because HMIPv6's hierarchical structure reduces the impact of mobility on the signaling cost. The signaling cost, therefore, decreases slower than that of SIGMA when MH's mobility decreases.

\section{Relative signaling cost of SIGMA to HMIPv6}

Fig. 4(d) shows the impact of (location update transmission cost) / (packet tunnelling cost) ratio $\left(\delta_{U} / \tau\right)$ on the relative sig- naling cost between SIGMA and HMIPv6. A higher $\delta_{U} / \tau$ ratio means that the location update requires more cost while packet encapsulation/decapsulation costs less. This ratio depends on the implementation of the intermediate routers. We can see that as long as $\delta_{U} / \tau<15$, the signaling cost of SIGMA is less than that of HMIPv6 due to the advantage of no tunnelling required by SIGMA. After that equilibrium point, the cost of location update dominates, and the signaling cost of SIGMA becomes higher than that of HMIPv6.

\section{Conclusions}

This paper evaluates the signaling cost of SIGMAby using an analytical model. Various aspects affecting SIGMA's signaling cost, such as mobile host moving speed, number of mobile host, number of correspondent node, per-hop transmission cost, and session to mobility ratio, have been considered. We also compared the signaling cost of SIGMA with that of HMIPv6. Numerical results show that, in most practical scenarios, the signaling cost of SIGMA is lower than HMIPv6. However, there is a tradeoff between location update transmission cost $\left(\delta_{U}\right)$ and packet tunnelling cost $(\tau)$; very high $\delta_{U} / \tau$ ratio results in the signaling cost of SIGMA being higher than that of HMIPv6.

\section{REFERENCES}

[1] C.E. Perkins (editor), "IP Mobility Support." IETF RFC 3344, August 2002.

[2] K.E. Malki (editor), "Low latency handoffs in Mobile IPv4." IETF DRAFT, draft-ietf-mobileip-lowlatency-handoffs-v4-07.txt, October 2003.

[3] R. Koodli (editor), "Fast handovers for Mobile IPv6." IETF DRAFT, draft-ietf-mipshop-fast-mipv6-03.txt, October 2004.

[4] H. Soliman, C. Catelluccia, and K.E. Malki et al., "Hierarchical Mobile IPv6 mobility management (HMIPv6)." IETF DRAFT, draft-ietfmipshop-hmipv6-04.txt, December 2004.

[5] R. Hsieh and A. Seneviratne, "A comparison of mechanisms for improving Mobile IP handoff latency for end-to-end TCP," ACM MobiCom, San Diego, USA, pp. 29-41, September 2003.

[6] S. Fu, L. Ma, M. Atiquzzaman, and Y. Lee, "Architecture and performance of SIGMA: A seamless mobility architecture for data networks," 40th IEEE Internationl Conference on Communications (ICC), Seoul, Korea, May 2005.

[7] J. Xie and I. F. Akyildiz, "An optimal location management scheme for minimizing signaling cost in Mobile IP," IEEE International Conference on Communications (ICC), New York, pp. 3313-3317, April 2002.

[8] Y.W. Chung, D.K. Sun, and A.H. Aghvami, "Steady state analysis of PMIP mobility management," IEEE Communication Letters, vol. 7, no. 6, pp. 278-280, June 2003.

[9] M.E. Crovella and A. Bestavros, "Self-similarity in world wide web traffic: evidence and possible causes," IEEE/ACM Transactions on Networking, vol. 5, no. 6, pp. 835-846, Dec 1997.

[10] V. Paxson and S. Floyd, "Wide area traffic: the failure of Poisson modeling," IEEE/ACM Transactions on Networking, vol. 3, no. 3, pp. 226-244, June 1995.

[11] C. Bettstetter, H. Hartenstein, and X. Perez-Costa, "Stochastic properties of the random waypoint mobility model: epoch length, direction distribution, and cell change rate," 5th ACM International Workshop on Modeling Analysis and Simulation of Wireless and Mobile Systems, pp. 7-14, September 2002.

[12] J. Xie and I. F. Akyildiz, "A novel distributed dynamic location management scheme for minimizing signaling costs in Mobile IP," IEEE Transactions on Mobile Computing, vol. 1, no. 3, pp. 163-175, July 2002.

[13] S. Fu, M. Atiquzzaman, L. Ma, and Y. Lee, "Signaling cost and performance of SIGMA: A seamless handover scheme for data networks," Accepted by Journal of Wireless Communications and Mobile Computing. 